Article

\title{
A Memristive Diode Bridge-Based Canonical Chua's Circuit
}

\section{Mo Chen, Jingjing Yu, Qing Yu, Changdi Li and Bocheng Bao *}

School of Information Science and Engineering, Changzhou University, Changzhou 213164, China; E-Mails: mchen@cczu.edu.cn (M.C); crystal_yu9011@126.com (J.Y.); 15161177256@163.com (Q.Y.); zby1819917975@163.com (C.L.)

* Author to whom correspondence should be addressed; E-Mail: mervinbao@126.com; Tel.: +86-519-8686-3883.

External Editor: J. A. Tenreiro Machado

Received: 30 September 2014; in revised form: 19 November 2014 / Accepted: 2 December 2014/ Published: 8 December 2014

\begin{abstract}
A novel memristor circuit is presented, which is generated from the canonical Chua's circuit by replacing the Chua's diode with a first order memristive diode bridge. The circuit dynamical characteristics with the variations of circuit parameters are investigated both theoretically and numerically. It can be found that the circuit has three determined equilibrium points, including a zero saddle point and two nonzero saddle-foci with index 2. Specially, the circuit is non-dissipative in the neighborhood of the zero saddle point, and there exists complex nonlinear phenomena of coexisting bifurcation modes and coexisting chaotic attractors. Experimental observations are performed to verify the numerical simulation results.
\end{abstract}

Keywords: coexisting bifurcation mode; Chua's circuit; memristive diode bridge; dynamical characteristics

PACS Codes: 05.45.-a

\section{Introduction}

Due to physical realizations of various memristors, much attention has been paid to memristor-based chaotic circuits and their dynamical analyses in recent years. Memristor-based chaotic circuits can be 
constructed by replacing nonlinear resistance elements in classic chaotic circuits with memristors, and some novel features of chaotic behaviors could be observed [1-12].

Since memristors are commercially unavailable, it would be very useful to have a circuit that emulates a memristor. Several nonlinearities are used to describe the relation between magnetic flux and electric charge of these memristors, such as HP memristor model [3,4,11,13], non-smooth piecewise linearity $[5,6]$, smooth cubic nonlinearity $[1,2,7,8]$, smooth piecewise-quadratic nonlinearity $[9,10]$, and so on. The corresponding equivalent circuits can easily be constructed using off-the-shelf components such as resistors, capacitors, operational amplifiers and analog multipliers, which are useful for memristor-based breadboard experiments.

Another approach is to use generalized memristors, which would require a circuit that can exhibit the desired memristor printfingers, such as memristive LDR circuit [14] and memristive diode bridge circuit $[15,16]$. A generalized memristor consisting of a diode bridge with a second order parallel RLC filter is reported in [15], which is further simplified by replacing the second order RLC filter with a first order parallel RC filter [16]. These memristive diode bridge-based circuits have simple topological structures, and only consist of elementary electronic circuit elements, which are suitable for using integrated circuit techniques, and can easily be used in other application circuits.

In this paper, a new memristive diode bridge-based chaotic circuit is proposed, which is constructed by replacing the Chua's diode in the canonical Chua's circuit with the simplified generalized memristor reported in [16]. In the proposed circuit, a physical circuit exhibiting memristor printfingers is used, which is different from memristive chaotic systems reported in [1-3,9-12], where the nonlinearities of memristors are described by mathematical models and special properties of line equilibrium set $[1-3,9,10]$, transient chaos [1,2] and hyperchaos [12] are discovered. Due to the nonlinearity of the memristive diode bridge, the proposed memristive Chua's circuit has three determined equilibrium points, including a zero unstable saddle point and two nonzero unstable saddle-foci with index 2. In particular, this circuit is non-dissipative in the neighborhood of the zero saddle point, and nonlinear phenomena of coexisting bifurcation modes and coexisting attractors are observed.

The dynamical characteristics of the proposed memristive Chua's circuit are thoroughly investigated using several traditional dynamical methods [1,2,9-12]. In Section 2, the mathematical model of the proposed circuit is deduced, and numerical results of state variables are calculated. According to simulation results, when proper circuit parameters are assigned, this circuit could be chaotic and displays a double-scroll chaotic attractor. In Section 3, the dissipativity of the memristive Chua's circuit is evaluated. Then the equilibrium points and their stabilities are analyzed to investigate the mechanism of its chaotic behaviors. In Section 4, the detailed dynamical behaviors are investigated by depicting its bifurcation diagrams and the corresponding Lyapunov exponent spectra. With reference to theoretical analysis results, typical phase portraits are numerically simulated and experimentally verified in Section 5.

\section{Mathematical Model of the Memristive Chua's Circuit}

The generalized memristor reported in [16] consists of a diode bridge with a first order parallel RC filter, as shown in Figure 1, whose memristive behavior is realized using the voltage constraints 
involving each pair of parallel diodes [15]. Its mathematical model is described by the following equation:

$$
\begin{gathered}
i=g\left(v_{C}, v\right) v=2 I_{S} e^{-\rho v_{C}} \sinh (\rho v) \\
\frac{\mathrm{d} v_{C}}{\mathrm{~d} t}=f\left(v_{C}, v\right)=\frac{2 I_{S} e^{-\rho v_{C}} \cosh (\rho v)}{C}-\frac{v_{C}}{R C}-\frac{2 I_{S}}{C}
\end{gathered}
$$

where $\rho=1 /\left(2 n V_{T}\right), I_{S}, n$, and $V_{T}$ stand for the reverse saturation current, emission coefficient, and thermal voltage of the diode, respectively, $v_{C}$ is the state variable of the dynamic element $C, v$ and $i$ are the input voltage and the flowing current of the generalized memristor. According to Equation (1), the generalized memristor is voltage-controlled and its memductance can be expressed by:

$$
G_{M}=\frac{i}{v}=g\left(v_{C}, v\right)=\frac{2 I_{S} e^{-\rho v_{C}} \sinh (\rho v)}{v}
$$

In this circuit, $C=1 \mu \mathrm{F}, R=0.5 \mathrm{k} \Omega$, and four $1 \mathrm{~N} 4148$ diodes are used to construct the memristive diode bridge, where the diode parameters are $I_{S}=2.682 \mathrm{nA}, n=1.836$, and $V_{T}=25 \mathrm{mV}$. When sinusoidal voltage stimuli are applied, the generalized memristor exhibits three characteristic fingerprints for identifying memristors [16].

Figure 1. Generalized memristor realized by memristive diode bridge with parallel RC filter [16].

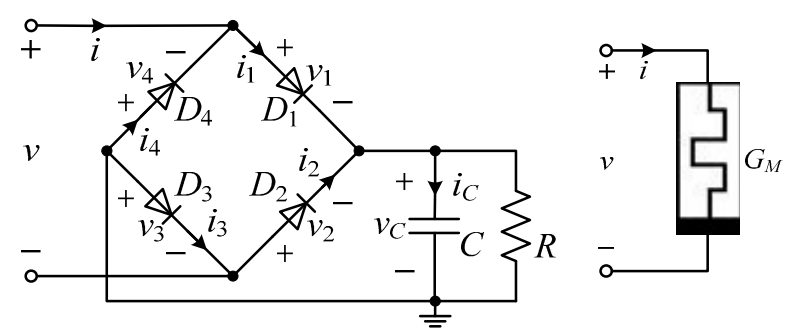

Based on the topology of the canonical Chua's circuit, a memristor based chaotic circuit is established, as shown in Figure 2, in which the Chua's diode is substituted by the simplified generalized memristor shown in Figure 1.

Figure 2. Generalized memristor-based canonical Chua's circuit.

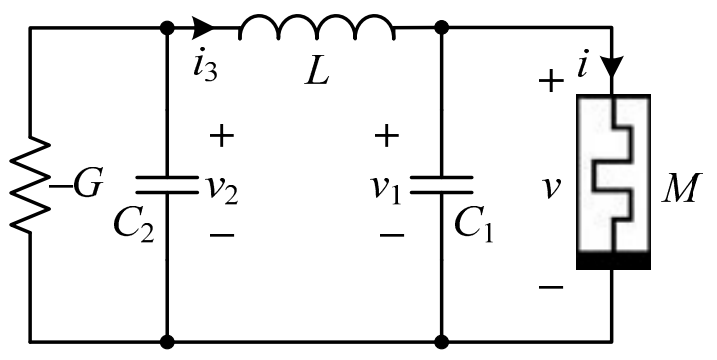

With reference to Figure 2, there are four dynamical elements of two capacitors $C_{1}$ and $C_{2}$, an inductor $L$, and the capacitor $C$ inside the generalized memristor, leading to the existence of four state variables $v_{1}, v_{2}, i_{3}$ and $v_{C}$. Applying Kirchhoff's circuit laws and the constitutive relationship of the generalized memristor of the proposed circuit, we can obtain a set of first-order differential equations: 


$$
\left\{\begin{aligned}
\frac{\mathrm{d} v_{1}}{\mathrm{~d} t} & =\frac{i_{3}}{C_{1}}-\frac{2 I_{S} e^{-\rho v_{C}} \sinh \left(\rho v_{1}\right)}{C_{1}} \\
\frac{\mathrm{d} v_{2}}{\mathrm{~d} t} & =\frac{G v_{2}}{C_{2}}-\frac{i_{3}}{C_{2}} \\
\frac{\mathrm{d} i_{3}}{\mathrm{~d} t} & =\frac{v_{2}}{L}-\frac{v_{1}}{L} \\
\frac{\mathrm{d} v_{C}}{\mathrm{~d} t} & =\frac{2 I_{S} e^{-\rho v_{C}} \cosh \left(\rho v_{1}\right)}{C}-\frac{v_{C}}{R C}-\frac{2 I_{S}}{C}
\end{aligned}\right.
$$

When the circuit parameters are fixed as listed in Table 1, and the initial values of four state variables are taken as $v_{1}(0)=0.01 \mathrm{~V}, v_{2}(0)=0.01 \mathrm{~V}, i_{3}(0)=0 \mathrm{~A}$ and $v_{C}(0)=0 \mathrm{~V}$, the circuit in Figure 2 is chaotic and displays a double-scroll chaotic attractor, as depicted in Figure 3a. The voltage-current relation of the generalized memristor is presented in Figure 3b, which exhibits a pinched hysteresis loop similar to that reported in [16]. This nonlinearity is important for the memristive Chua's circuit to generate special dynamical behaviors showing in next sections. The corresponding Poincaré mapping on $v c(t)=0.5505 \mathrm{~V}$ section is illustrated in Figure 3c, which further prove that the circuit is indeed chaotic.

Figure 3. Dynamics of generalized memristor based canonical Chua's circuit. (a) Phase portrait in $v_{1}(t)-v_{2}(t)$ plane; (b) Phase portrait in $v_{1}(t)-i(t)$ plane and (c) Poincaré mapping in $v_{1}(t)-v_{2}(t)$ plane.

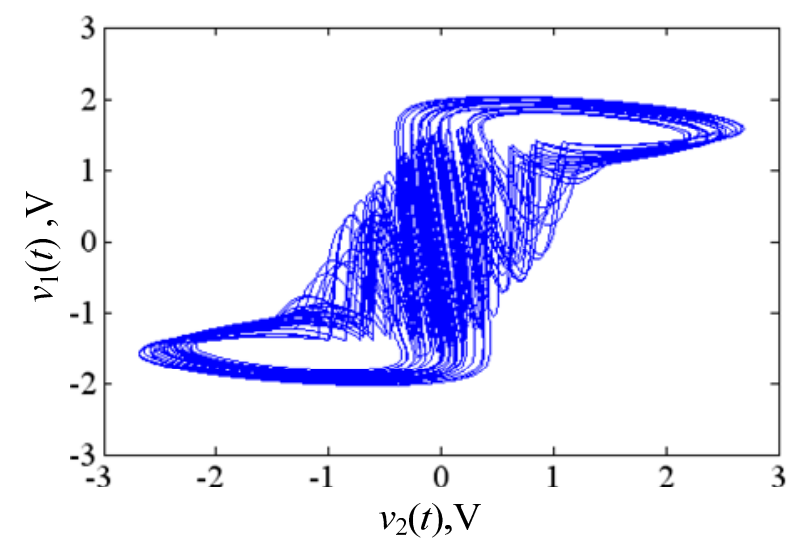

(a)

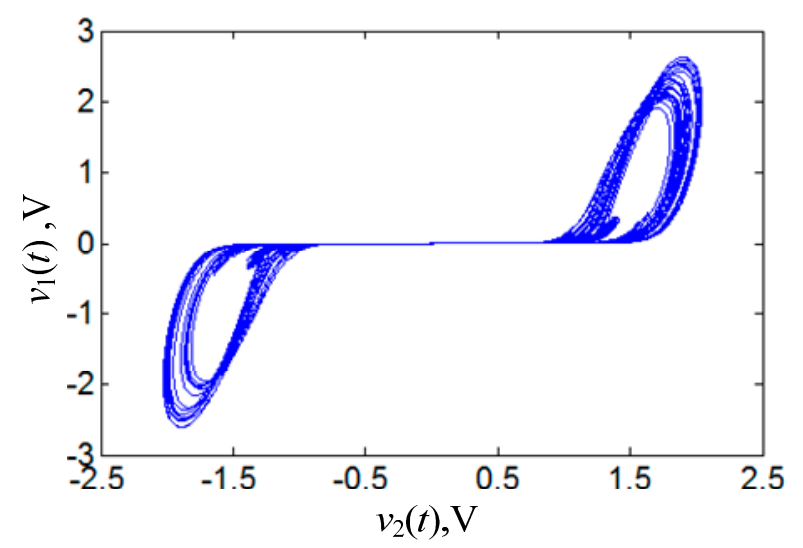

(b)

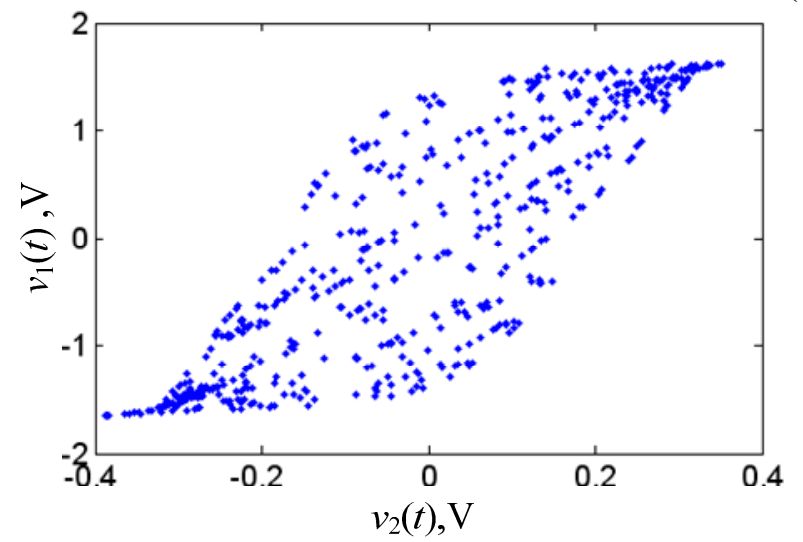

(c) 


\section{Equilibrium Points and Stabilities}

The concept of dissipativity for linear and nonlinear systems was introduced by Willems in $1972[17,18]$. It is well known that a nonlinear circuit is dissipative if it has a minus exponential constrain rate, then the orbits of this circuit will ultimately be confined to a specific sub-set of zero volume, and the asymptotic motion will settle onto an attractor [19,20]. For the circuit in Figure 2, the mathematical expression of the exponential constrain rate is written as:

$$
\nabla V=\frac{\partial \dot{v}_{1}}{\partial v_{1}}+\frac{\partial \dot{v}_{2}}{\partial v_{2}}+\frac{\partial \dot{i}_{3}}{\partial \dot{i}_{3}}+\frac{\partial \dot{v}_{C}}{\partial v_{C}}=-2 \rho I_{S} e^{-\rho v_{C}}\left[\frac{\cosh \left(\rho v_{1}\right)}{C_{1}}+\frac{\cosh \left(\rho v_{2}\right)}{C}\right]+\frac{G}{C_{2}}-\frac{1}{R C}
$$

Table 1. Circuit parameters for simulations and experiments.

\begin{tabular}{ccc}
\hline Parameters & Significations & Values \\
\hline$R$ & Resistance & $0.5 \mathrm{k} \Omega$ \\
$G$ & Conductance & $0.67 \mathrm{mS}$ \\
$L$ & Inductance & $185 \mathrm{mH}$ \\
$C$ & Capacitance & $1 \mu \mathrm{F}$ \\
$C_{1}$ & Capacitance & $0.02 \mu \mathrm{F}$ \\
$C_{2}$ & Capacitance & $0.2 \mu \mathrm{F}$ \\
$I_{S}$ & reverse saturation current of diode & $2.682 \mathrm{nA}$ \\
$n$ & emission coefficient of diode & 1.836 \\
$V_{T}$ & thermal voltage of diode & $25 \mathrm{mV}$ \\
\hline
\end{tabular}

Using circuit element parameters listed in Table 1, Equation (5) can be written as following:

$$
\nabla V=-2.92 e^{-\rho v_{C}}\left[\cosh \left(\rho v_{1}\right)+\frac{\cosh \left(\rho v_{2}\right)}{50}\right]+1330.4
$$

According to Equation (6), the dissipativity of the proposed circuit is determined by the values of state variables $v_{1}(t), v_{2}(t)$ and $v_{\mathrm{C}}(t)$. Obviously, the circuit is non-dissipative in the region around the origin point, but chaotic attractor can still be generated, as shown in Figure 3. This property is different from conventional nonlinear chaotic circuits [19,20], which are complete dissipative for the selected circuit element parameters, so the eigenvalues of the corresponding Jacobian matrix at each equilibrium points should be evaluated to reveal the mechanism of the chaotic behavior illustrated in Figure 3.

Firstly, the equilibrium points of the circuit in Figure 2 are calculated by setting the right-hand side of Equation (4) to zero, and we can obtain the following equations:

$$
\left\{\begin{array}{rl}
i_{3} & =2 I_{S} e^{-\rho v_{C}} \sinh \left(\rho v_{1}\right) \\
i_{3} & =G v_{2} \\
v_{2} & =v_{1} \\
v_{C} & =2 R I_{S} e^{-\rho v_{C}} \cosh \left(\rho v_{1}\right)-2 R I_{S}
\end{array} .\right.
$$

Clearly, the origin point $S_{0}=(0,0,0,0)$ is one equilibrium point of the memristive circuit. Besides, two functions describing the relationship between $v_{C}$ and $v_{1}$ are also derived as: 


$$
\begin{gathered}
v_{C}=f_{1}\left(v_{1}\right)=\frac{1}{\rho} \ln \frac{2 I_{S} \sinh \left(\rho v_{1}\right)}{G v_{1}}, \\
v_{C}=f_{2}\left(v_{1}\right)=R G v_{1} \operatorname{coth}\left(\rho v_{1}\right)-2 R I_{S} .
\end{gathered}
$$

The values of $v_{C}$ and $v_{1}$ are the intersection points of these two function curves described by Equations (8) and (9), which can be obtained through graphic analytic method as shown in Figure 4. According to the lines depicted in Figure 4 and the circuit parameters listed in Table 1, it is easily verified that the proposed chaotic circuit has two nonzero equilibrium points:

$$
\begin{aligned}
& S_{1}=(1.7909,1.7909,0.0012,0.5970) \\
& S_{2}=(-1.7909,-1.7909,-0.0012,0.5970)
\end{aligned}
$$

These two equilibrium points are located symmetrically on both sides of the $v_{C}$-axis, whose values are determined by the specified circuit parameters of $G$ and $R$. This feature is completely different from conventional memristor based chaotic circuits $[1-3,9,10]$, whose equilibrium points are an equilibrium point set located on the axis corresponding to the inner state variable of the memristor.

Figure 4. Two function curves and their intersection points.

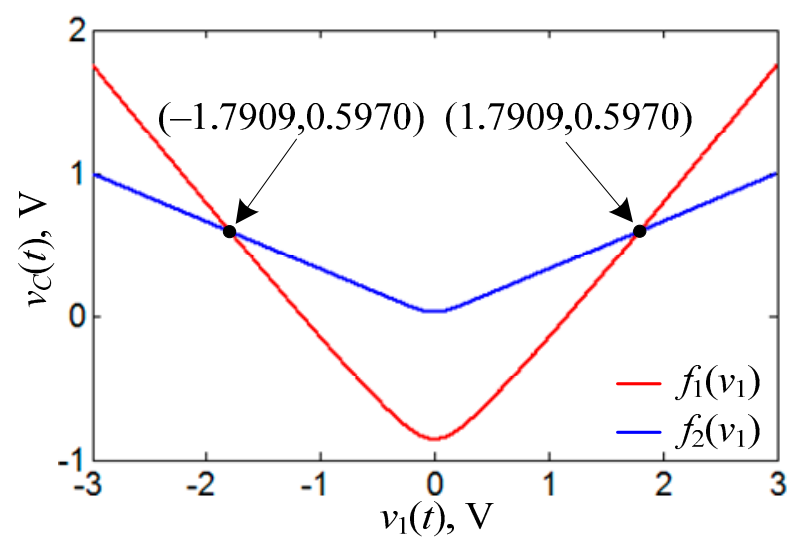

Then, the Jacobian matrix, evaluated at the equilibrium point $\bar{S}=\left(\bar{v}_{1}, \bar{v}_{2}, \bar{i}_{3}, \bar{v}_{C}\right)$, is given by:

$$
\mathbf{J}=\left[\begin{array}{cccc}
-\frac{h_{2}}{C_{1}} & 0 & \frac{1}{C_{1}} & \frac{h_{1}}{C_{1}} \\
0 & \frac{G}{C_{2}} & -\frac{1}{C_{2}} & 0 \\
-\frac{1}{L} & \frac{1}{L} & 0 & 0 \\
\frac{h_{1}}{C} & 0 & 0 & -\frac{h_{2}}{C}-\frac{1}{R C}
\end{array}\right]
$$

where, $h_{1}=2 \rho I_{S} e^{-\rho \bar{v}_{C}} \sinh \left(\rho \bar{v}_{1}\right)$ and $h_{2}=2 \rho I_{S} e^{-\rho \bar{v}_{C}} \cosh \left(\rho \bar{v}_{1}\right)$. Thus, the characteristic equation of Equation (11) is written as:

$$
\lambda^{4}+a_{3} \lambda^{3}+a_{2} \lambda^{2}+a_{1} \lambda+a_{0}=0
$$

where: 


$$
\begin{aligned}
& a_{3}=\frac{h_{2}}{C}+\frac{h_{2}}{C_{1}}+\frac{1}{R C}-\frac{G}{C_{2}} \\
& a_{2}=\frac{C_{1}+C_{2}}{L C_{1} C_{2}}-\frac{h_{1}^{2}-h_{2}^{2}}{C C_{1}}-\frac{G}{C C_{2}}\left(h_{2}+\frac{1}{R}\right)+\frac{h_{2}}{C_{1}}\left(\frac{1}{R C}-\frac{G}{C_{2}}\right) \\
& a_{1}=\frac{1}{C_{1} C_{2}}\left[\frac{G h_{1}^{2}}{C}+\frac{h_{2}-G}{L}+\left(\frac{h_{2}}{C}+\frac{1}{R C}\right)\left(\frac{C_{1}+C_{2}}{L}-G h_{2}\right)\right] \\
& a_{0}=\frac{1}{L C C_{1} C_{2}}\left[\left(h_{2}-G\right)\left(h_{2}+\frac{1}{R}\right)-h_{1}^{2}\right]
\end{aligned}
$$

Finally, four eigenvalues at three determined equilibrium points are calculated as:

$$
\begin{array}{ll}
S_{0}: & \lambda_{1}=3039.2, \lambda_{2,3}=145.6 \pm \mathrm{j} 17215.7, \lambda_{4}=-2000.1 \\
S_{1,2}: & \lambda_{1,2}=1073.3 \pm \mathrm{j} 5163.3, \lambda_{3}=-1173.3, \lambda_{4}=-663303.0
\end{array}
$$

It can be seen that $S_{0}$ is a unstable saddle point having a positive real root, two complex conjugate roots with positive real parts, and a negative real root; whereas $S_{1,2}$ are two unstable saddle-foci having two complex conjugate roots with positive real part and two negative real roots. The exponential constrain rates at these equilibria are given as:

$$
\begin{aligned}
& \nabla V_{S_{0}}=1330.4 \\
& \nabla V_{S_{1,2}}=-662326.8
\end{aligned}
$$

With reference to Equation (15), the memristive Chua's circuit is non-dissipative in the neighborhood of $S_{0}$. Considering that $S_{0}$ is a repulsive point, the orbit will be excluded from this region, and ultimately settles onto an attractor around the two unstable saddle-foci in dissipative region. According to this, the proposed memristive Chua's circuit has two separated attractive regions, resulting to the formation of a double-scroll chaotic attractor, as depicted in Figure 3.

\section{Complex Dynamics in the Memristive Chaotic Circuit}

In this section, detailed dynamical behaviors of the proposed memristive circuit are analyzed by utilizing several conventional dynamical methods [1,2,9-12], in which the circuit parameters are set as illustrated in Table 1, and $L$ is selected as a varying parameter.

\subsection{Parameter Region}

According to Routh-Hurwutz condition, the real parts of the roots of Equation (12) are negative if and only if:

$$
a_{3}>0, a_{3} a_{2}-a_{1}>0 \text { and } a_{3}\left(a_{1} a_{2}-a_{0} a_{3}\right)-a_{1}^{2}>0, a_{0}>0
$$

For $S_{0}, a_{0}$ is always negative, which proves that $S_{0}$ is an unstable point. With reference to $S_{1}$ and $S_{2}$, Equation (16) gives rise to the following constraint condition:

$$
L<30.4431 \mathrm{mH}
$$


To make the nonzero equilibrium points unstable, thereby yielding the possibility for chaos to occur, the parameter $L$ needs to satisfy the following relation:

$$
L>30.4431 \mathrm{mH}
$$

So, the proposed memristive circuit is analyzed in the region $50 \mathrm{mH} \leq L \leq 300 \mathrm{mH}$, where chaotic behaviors could be observed. As $L$ increases gradually within the parameter variation range, the locations of the two nonzero equilibrium points are fixed, but their eigenvalues are changing, as presented in Table 2. It can be found that $S_{1,2}$ are two unstable saddle-foci having two conjugate roots with positive real parts and two negative real roots, from which a double-scroll chaotic attractor could be emerged. The detailed dynamical characteristics are investigated in next section through numerical simulations.

Table 2. Four eigenvalues $\lambda_{i}(i=1,2,3,4)$ of the nonzero equilibrium points.

\begin{tabular}{cccc}
\hline $\boldsymbol{L}$ & $\boldsymbol{\lambda}_{\mathbf{1 , 2}}$ & $\boldsymbol{\lambda}_{\mathbf{3}}$ & $\boldsymbol{\lambda}_{\mathbf{4}}$ \\
\hline $50 \mathrm{mH}$ & $484.1 \pm \mathrm{j} 10593.9$ & $-1,075.3$ & $-662,222.8$ \\
$160 \mathrm{mH}$ & $1033.1 \pm \mathrm{j} 5620.5$ & $-1,155.4$ & $-663,240.8$ \\
$220 \mathrm{mH}$ & $1117.4 \pm \mathrm{j} 4653.6$ & $-1,197.9$ & $-663,366.8$ \\
$300 \mathrm{mH}$ & $1189.3 \pm \mathrm{j} 3827.8$ & $-1,252.2$ & $-663,456.4$ \\
\hline
\end{tabular}

\subsection{Bifurcation Diagram and Lyapunov Exponents}

The bifurcation diagrams of $v_{2}(t)$ and the first three Lyapunov exponents $\mathrm{LE}_{1}, \mathrm{LE}_{2}$ and $\mathrm{LE}_{3}$ are depicted in Figure 5a,b, respectively. When $L$ increases gradually within the parameter region, the orbits of the memristive Chua's circuit start from periodic behavior, and then enter into chaotic behavior via forward period-doubling bifurcation routes. After this, the orbits return to periodic behavior through reverse period-doubling bifurcation routes, and then jump into chaotic behavior, and finally turn into infinite. Correspondingly, the maximum Lyapunov exponent equals zero in periodic regions, and reaches at positive during the chaotic regions. The numerical results showing in Figure 5a,b match well with each other.

Figure 5. Dynamics with the value of $L$ increasing. (a) Bifurcation diagram of $v_{2}(t)$ and (b) Lyapunov exponent spectra.

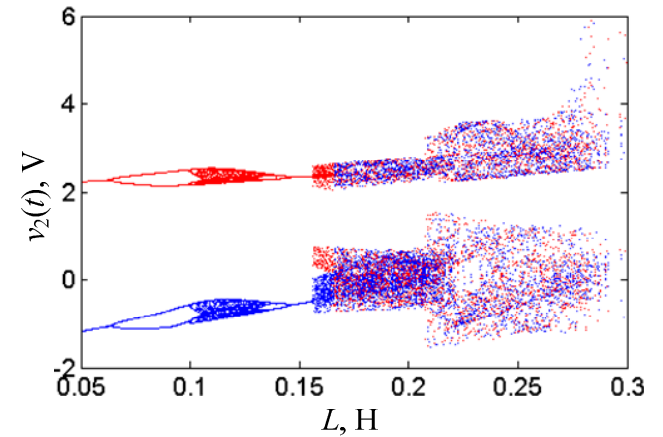

(a)

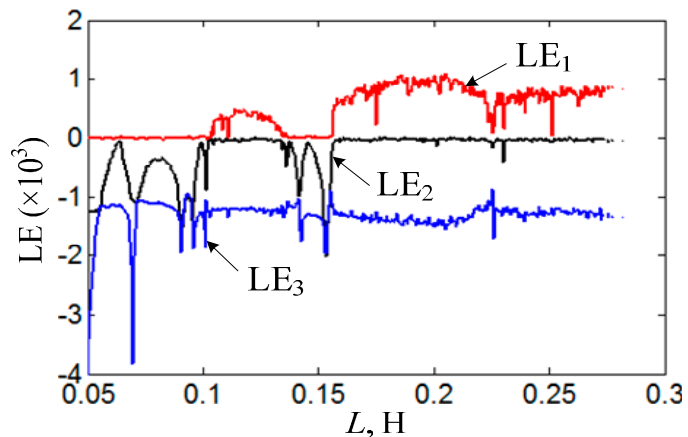

(b)

From Figure 5a, dynamical behavior of coexisting bifurcation mode is also observed, which mainly happened in the region $50 \mathrm{mH} \leq L \leq 165 \mathrm{mH}$. The trajectory colored in red starts with an initial 
condition of $[0.01 \mathrm{~V}, 0.01 \mathrm{~V}, 0 \mathrm{~A}, 0 \mathrm{~V}]$, whereas the one colored in blue starts with an initial condition of $[0.01 \mathrm{~V},-0.01 \mathrm{~V}, 0 \mathrm{~A}, 0 \mathrm{~V}]$.

Based on the eigenvalues illustrated in Table 2, when $L$ is increasing, the attraction and repulsion of $S_{1}$ and $S_{2}$ are getting stronger, while the increase of their repulsion is much larger. Then, the two separated attractive regions are gradually expanding, and merge into a double-scroll chaotic attractor as $L$ is greater than $165 \mathrm{mH}$.

\subsection{Coexisting Limit Cycles}

With reference to Figure $5 \mathrm{~b}$, when $50 \mathrm{mH} \leq L \leq 103 \mathrm{mH}$ and $134 \mathrm{mH} \leq L \leq 156 \mathrm{mH}$, the first Lyapunov exponent equals zero and the others are less than zero, that implies the existence of limit cycles with different periodicities. Some phase portraits in $v_{1}(t)-v_{2}(t)$ plane are obtained from numerical simulations and plotted in Figure $6 \mathrm{a}, \mathrm{b}$, in which $L$ is set to be $55 \mathrm{mH}$ and $85 \mathrm{mH}$, respectively. It can be found that there are two coexisting limit cycles located around the two nonzero equilibrium points. According to their geometric locations, the orbit colored in red is called the right limit cycle with the initial states $[0.01 \mathrm{~V}, 0.01 \mathrm{~V}, 0 \mathrm{~A}, 0 \mathrm{~V}]$, while the one colored in blue is called the left limit cycle with the initial states $[0.01 \mathrm{~V},-0.01 \mathrm{~V}, 0 \mathrm{~A}, 0 \mathrm{~V}]$. The time-domain waveforms of $v_{2}(t)$ at $L=85 \mathrm{mH}$, starting from the above mentioned different initial states, are plotted in Figure 7a. When the initial states are set close to equilibrium point $S_{1}$, the trajectory will be centered in the positive region of $v_{2}(t)$, otherwise the trajectory will be centered in the negative region of $v_{2}(t)$.

Figure 6. Chaotic and periodic orbits in $v_{1}(t)-v_{2}(t)$ plane. (a) Coexisting limit cycles with period-1 $(L=55 \mathrm{mH})$; (b) coexisting limit cycles with period-2 $(L=85 \mathrm{mH})$; (c) coexisting spiral chaotic attractors $(L=160 \mathrm{mH})$ and $(\mathbf{d})$ double-scroll chaotic attractor $(L=220 \mathrm{mH})$.

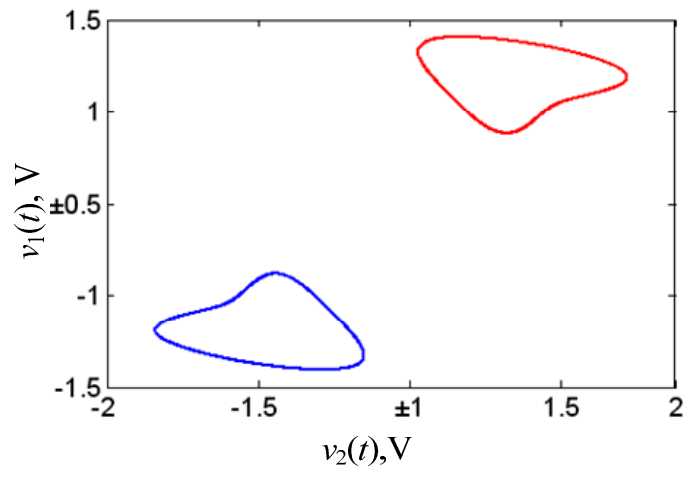

(a)

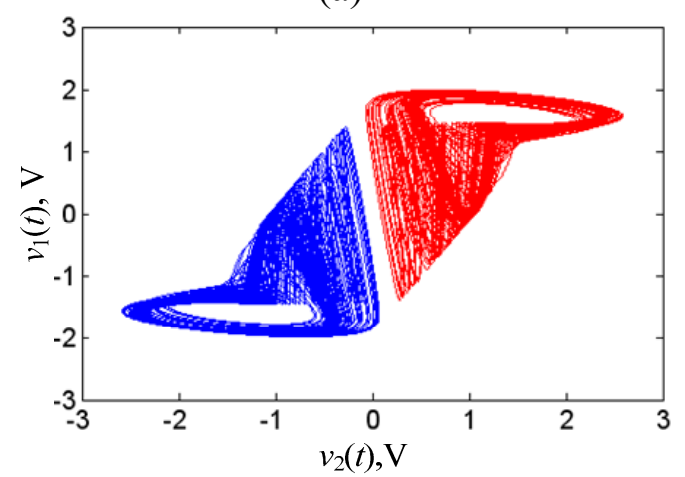

(c)

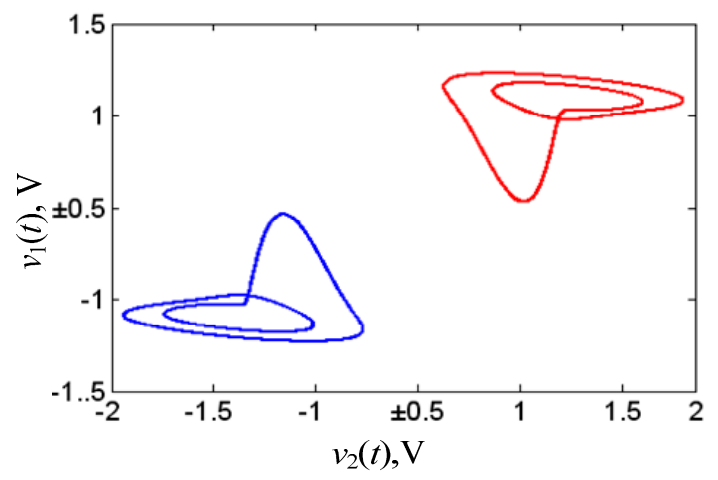

(b)

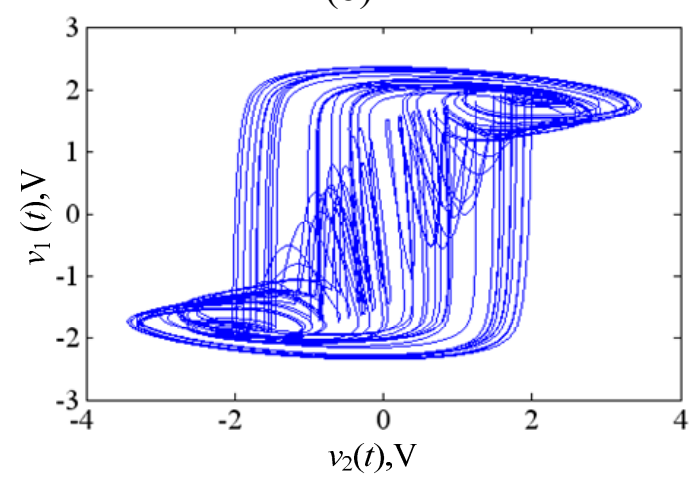

(d) 


\subsection{Coexisting Chaotic Attractors}

When $L$ is in the regions [104 $\mathrm{mH}, 132 \mathrm{mH}$ ] and [157 mH, $165 \mathrm{mH}$ ], the first Lyapunov exponent is positive, the second one equals zero, and the others are still less than zero, that implies the circuit in Figure 2 is chaotic. Figure $6 \mathrm{c}$ shows a phase portrait in $v_{1}(t)-v_{2}(t)$ plane at $L=160 \mathrm{mH}$, from which dynamical behaviors of coexisting chaotic attractors are observed. When the initial states are set close to equilibrium point $S_{1}$, the attractor colored in red will be emerged, which could be called as the right attractor. Otherwise, the left attractor colored in blue will be formed. The corresponding time-domain waveforms of $v_{2}(t)$ are similar to those depicted in Figure $7 \mathrm{a}$.

Figure 7. Time-domain waveforms of $v_{2}(t)$. (a) $L=85 \mathrm{mH}$ and (b) $L=185 \mathrm{mH}$.

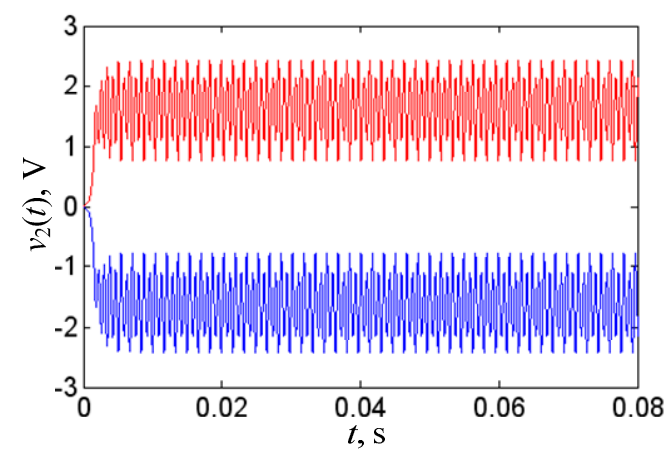

(a)

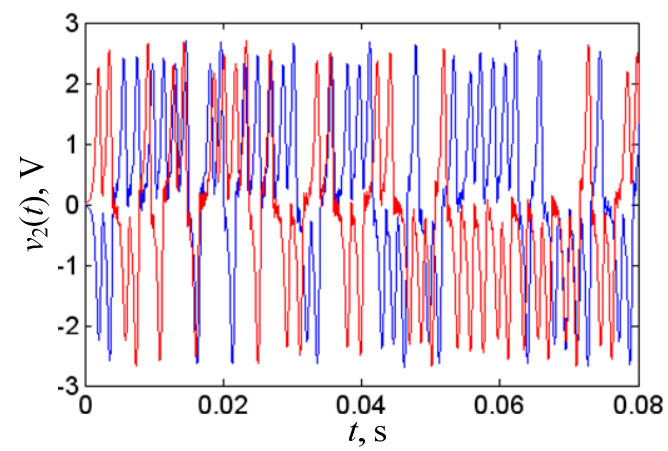

(b)

\subsection{Double-Scroll Chaotic Attractors}

As $L$ is increased, the two separated attractive regions illustrated in Figures $6 \mathrm{a}-\mathrm{c}$ are expanded, and the gap between these two regions gradually disappears. When $L$ is greater than $165 \mathrm{mH}$, these two orbits can pass through the boundary between right and left attractive domains, and a double-scroll attractor could be formed, as illustrated in Figure 6d, in which $L$ is set as $220 \mathrm{mH}$. The corresponding time-domain waveforms of $v_{2}(t)$ are plotted in Figure $7 \mathrm{~b}$. Obviously, the two trajectories span both negative and positive regions of $v_{2}(t)$.

When $L$ is further increased, the repulsion forces of $S_{1}$ and $S_{2}$ are getting too much stronger, and the chaotic orbits will escape from the attractive region to infinite as $L$ is greater than $278 \mathrm{mH}$.

\section{Experimental Verifications}

In this section, basic characteristics of complex dynamics of the proposed chaotic circuit are verified by experimental observations. An analog electronic circuit can easily been built to physically realize the chaotic circuit for generating chaotic attractor, in which precision potentiometers, manually winding inductors, multilayer ceramic capacitors and 1N4148 diodes are used. The negative conductor is realized by a current inverter with an $\mathrm{AD} 711 \mathrm{KN}$ operational amplifier and three resistors [9].

Setting the component parameters as those used in numerical simulations, the desired phase portraits are captured using Tektronix DPO3034 digital oscilloscope and depicted in Figure 8. Comparing Figure 8 with Figure 6, it can be seen that the shape of the measured phase portraits are slightly different from those obtained from numerical simulations, which may be caused by 
measurement errors arose from inaccurate values of manually winding inductor and parasitic parameters in the experimental circuit. But the dynamical behaviors and their evolution route observed from the analog electronic circuit agree with those revealed by numerical simulations. Therefore, it can be concluded that the experimental results can verify the results of theoretical analyses and numerical simulations.

Figure 8. Experimental results of phase portraits in $v_{1}(t)-v_{2}(t)$ plane. (a) Coexisting limit cycles with period-1 $(L=55 \mathrm{mH})$; (b) coexisting limit cycles with period-2 $(L=85 \mathrm{mH})$; (c) coexisting spiral chaotic attractors $(L=160 \mathrm{mH})$ and (d) double-scroll chaotic attractor $(L=220 \mathrm{mH})$.

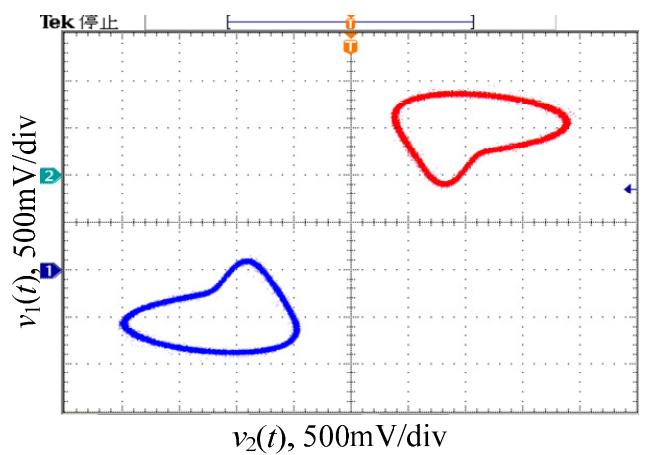

(a)

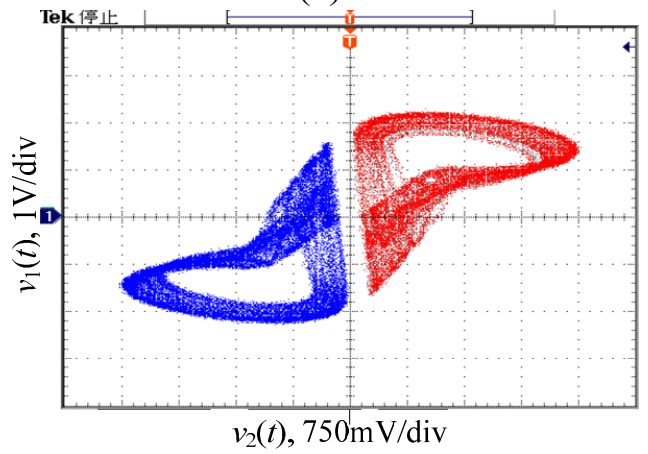

(c)

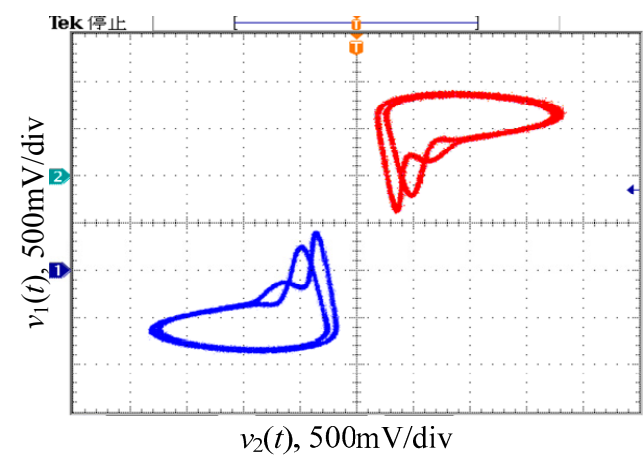

(b)

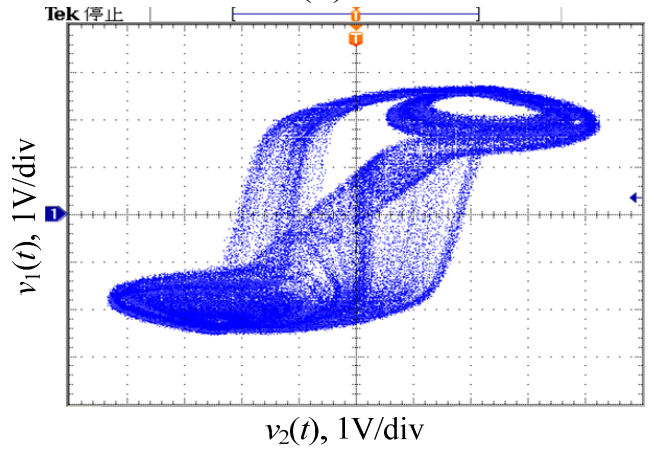

(d)

\section{Conclusions}

In this paper, a novel memristor based chaotic circuit is presented, which is derived from the canonical Chua's circuit by replacing the Chua's diode with a first order memristive diode bridge. The equilibrium points and their stabilities are analyzed, and the dynamical characteristics, with the variation of parameter $L$, are investigated both theoretically and numerically. The simulation results indicate that this circuit has three determined equilibrium points, one zero unstable saddle point and two symmetrical unstable saddle-foci. Specially, the proposed memristive circuit is non-dissipative in the neighborhood of the zero equilibrium point, but chaotic attractors excited from two nonzero unstable saddle-foci could still be observed. For special properties of these equilibria, nonlinear phenomena of coexisting bifurcation modes and coexisting attractors are also discovered. Finally, a simple electronic circuit is realized and some chaotic attractors are obtained. The dynamical behaviors of the analog electronic circuit agree with those revealed by numerical simulation results. 


\section{Acknowledgments}

This work is supported by the grants from the National Natural Science Foundation of China (Grant No. 51277107) and the Natural Science Foundation of Jiangsu Province, China (Grant No. BK2012583).

\section{Author Contributions}

Bocheng Bao and Mo Chen were responsible for the conception and design of manuscript. Jingjing $\mathrm{Yu}$ performed numerical simulations and analyzed the data. Qing $\mathrm{Yu}$ and Changdi Li were responsible for experimental validation. All authors have read and approved the final manuscript.

\section{Conflicts of Interest}

The authors declare no conflict of interest.

\section{Reference}

1. Bao, B.C.; Ma, Z.H.; Xu, J.P.; Liu, Z.; Xu,Q. A simple memristor chaotic circuit with complex dynamics. Int. J. Bifurc. Chaos 2011, 21, 2629-2645.

2. Bao, B.C.; Liu, Z.; Xu, J.P. Steady periodic memristor oscillator with transient chaotic behaviors. Electron. Lett. 2010, 46, 228-230.

3. Wang, G.Y.; He, J.L.; Yuan, F.; Peng, C.J. Dynamical behaviors of a $\mathrm{TiO}_{2}$ memristor oscillator. Chin. Phys. Lett. 2013, 30, 110506.

4. Wang, L.; Drakakis, E.; Duan, S.; He, P.; Liao, X. Memristor model and its application for chaos generation. Int. J. Bifurc. Chaos 2012, 22, 1250205.

5. Muthuswamy, B.; Kokate, P.P. Memristor-based chaotic circuits. IETE Tech. Rev. 2009, 26, 415-426.

6. Itoh, M.; Chua, L.O. Memristor oscillators. Int. J. Bifurc. Chaos 2008, 18, 3183-3206.

7. Muthuswamy, B. Implementing memristor based chaotic circuits. Int. J. Bifurc. Chaos 2010, 20, 1335-1350.

8. Iu, H.H.C.; Yu, D.S.; Fitch, A.L.; Sreeram, V.; Chen, H. Controlling chaos in a memristor based circuit using a twin-T notch filter. IEEE Trans. Circuits Syst. I 2011, 58, 1337-1344.

9. Bao, B.C.; Xu J.P.; Zhou, G.H.; Ma, Z.H.; Zou, L. Chaotic memristive circuit: equivalent circuit realization and dynamical analysis. Chin. Phys. B 2011, 20, 120502.

10. Bao, B.C.; Xu, J.P.; Liu, Z. Initial state dependent dynamical behaviors in memristor based chaotic circuit. Chin. Phys. Lett. 2010, 27, 070504.

11. Wang W.; Wang, G.Y.; Wang, X.Y. Research on a chaotic circuit based on an active $\mathrm{TiO}_{2}$ memristor. Syst. Sci. Contr. Eng. 2014, doi:10.1080/21642583.2013.879842.

12. Fitch, A.L.; Yu, D.; Iu, H.H.C.; Sreeram, V. Hyperchaos in a memristor-based modified canonical Chua's circuit. Int. J. Bifurc. Chaos 2012, 22, 1250133.

13. Kim, H.; Sah, M.P.; Yang, C.J.; Cho, S.; Chua, L.O. Memristor emulator for memristor circuit applications. IEEE Trans. Circuits Syst. I 2012, 59, 2422-2431. 
14. Wang, X.Y.; Fitch, A.L.; Iu, H.H.; Sreeram, V.; Qi, W.G. Implementation of an analogue model of a memristor based in a light-dependent resistor. Chin. Phys. B 2012, 21, 108501.

15. Corinto, F.; Ascoli, A. Memristive diode bridge with LCR filter. Electron. Lett. 2012, 48, 824-825.

16. Bao, B.C.; Yu, J.J.; Hu, F.W. Generalized memristor consisting of diode bridge with first order parallel RC filter. Int. J. Bifurc. Chaos 2014, 24, 1450143.

17. Willems, J.C. Dissipative dynamical systems part I: General theory. Arch. Ration. Mech. Anal. 1972, 45, 321-351.

18. Willems, J.C. Dissipative dynamical systems part II: Linear systems with quadratic supply rates. Arch. Ration. Mech. Anal. 1972, 45, 352-393.

19. Dadras, S.; Momeni, H.R.; Qi, G. Analysis of a new 3D smooth autonomous system with different wing chaotic attractors and transient chaos. Nonlinear Dyn. 2010, 62, 391-405.

20. Sprott, J.C.; Wang, X.; Chen, G. Coexistence of point, periodic and strange attractors. Int. J. Bifurc. Chaos 2013, 23, 1350093.

(C) 2014 by the authors; licensee MDPI, Basel, Switzerland. This article is an open access article distributed under the terms and conditions of the Creative Commons Attribution license (http://creativecommons.org/licenses/by/4.0/). 